EL COMPORTAMIENTO DE LAS CATEGORÍAS GRAMATICALES Y LA PRECISIÓN LÉXICA EN TEXTOS ORALES NARRATIVOS Y EXPLICATIVOS PRODUCIDOS POR ESCOLARES EN COSTA RICA

THE BEHAVIOR OF THE GRAMMAR CATEGORIES AND LEXICAL PRECISION ON NARRATIVE AND EXPLANATORY ORAL TEXT PRODUCED BY STUDENTS IN COSTA RICA

Volumen 11, Número 3

Setiembre-Diciembre pp. 1-25

Este número se publicó el 15 de diciembre de 2011

Jessica Araya Ramírez

La revista está indexada en los directorios:

LATINDEX, REDALYC, IRESIE, CLASE, DIALNET, DOAJ, E-REVIST@S,

La revista está incluida en los sitios:

REDIE, RINACE, OEI, MAESTROTECA, PREAL, HUASCARAN, CLASCO 


\title{
EL COMPORTAMIENTO DE LAS CATEGORÍAS GRAMATICALES Y LA PRECISIÓN LÉXICA EN TEXTOS ORALES NARRATIVOS Y EXPLICATIVOS PRODUCIDOS POR ESCOLARES EN COSTA RICA \\ THE BEHAVIOR OF THE GRAMMAR CATEGORIES AND LEXICAL PRECISION ON NARRATIVE AND EXPLANATORY ORAL TEXT PRODUCED BY \\ STUDENTS IN COSTA RICA
}

\begin{abstract}
Jessica Araya Ramírez ${ }^{1}$
Resumen: Este es un artículo que parte de una investigación realizada en una escuela pública de la Dirección Regional de San José, circuito 10, en el año 2010, en la cual se analizó el comportamiento de las categorías gramaticales; sustantivos, adjetivos y verbos, se utilizó la Prueba de diversidad léxica en textos de cincuenta enunciados Pd50, así como la precisión léxica presente en los textos orales narrativos y explicativos de treinta y seis escolares. Los resultados arrojados reflejan el poco uso de los adjetivos y una diversidad de imprecisiones léxicas que obstaculizaron una adecuada comprensión del mensaje, por lo que es necesaria una intervención didáctica oportuna que permita que los escolares utilicen el vocabulario en forma variada y precisa.
\end{abstract}

Palabras clave: EDUCACIÓN PRIMARIA, VOCABULARIO, ESCOLARES, PRECISIÓN LÉXICA, APRENDIZAJE, COSTA RICA

\begin{abstract}
This article is part of an investigation into a public school in the Regional Office in San Jose, circuit 10, in the year 2010, which analyzed the behavior of parts of speech, nouns, adjectives and verbs used Proof of lexical diversity in texts of fifty statements PD50 and lexical accuracy present in oral texts and explanatory narrative of thirty-six schools. The results shown reflect the limited use of adjectives and a variety of lexical inaccuracies that prevented a proper understanding of the message, so it's timely intervention necessary teaching that allows students to use vocabulary in a varied and precise.
\end{abstract}

Keywords: PRIMARY-SCHOOL, VOCABULARY, SCHOOL, LEARNING-LEXICAL, PRECISION, COSTA RICA

\footnotetext{
${ }^{1}$ Máster en Lingüística de la Universidad de Costa Rica. Profesora de la Escuela de Formación Docente e investigadora del Instituto de Investigación en Educación (INIE) de la Universidad de Costa Rica.
}

Dirección electrónica: jesaraya30@yahoo.es

Artículo recibido: 10 de agosto, 2011

Aprobado: 28 de noviembre, 2011 


\section{Introducción}

El objetivo general de la educación lingüística enfatiza la adquisición de las capacidades comunicativas para comprender y generar discursos adecuados para cada contexto comunicativo, meta que se operacionaliza en el ambiente escolar mediante la promoción de las habilidades lingüísticas: hablar, escuchar, leer, comprender y escribir.

Al respecto, Lomas (1999) señala que los objetivos de la comunicación oral buscan el logro de una expresión coherente, adecuada al contexto, respetuosa de los aspectos normativos de la lengua, y que combine recursos lingüísticos y no lingüísticos para producir e interpretar mensajes con diferentes intenciones comunicativas que atiendan las pautas propias de ese intercambio.

En este sentido, la promoción de la producción oral, es decir, ese conjunto de habilidades, destrezas, conocimientos y actitudes lingüísticas necesarias para desempeñarse en forma adecuada dentro del plano comunicativo, requerirá de la programación y planificación sistematizada -en el aula- de formas discursivas espontáneas y posteriormente de modalidades expresivas discursivas más formales en las que las competencias puedan ser adquiridas y ejercitadas en forma sistemática.

En cuanto a esto, se asume en ocasiones que los niños al ingresar a la escuela ya saben hablar y, por lo tanto, no es necesaria una mediación pedagógica que norme y permita acrecentar las habilidades lingüísticas.

Asimismo, en la educación costarricense, las producciones orales de los niños son orientadas, generalmente, hacia la preparación y el desarrollo de exposiciones de temas presentes en los programas escolares. Este tipo de actividad debilita los objetivos de la expresión oral dado que el interés del educador es el dominio del tema, el cual se limita a la memorización o lectura de los datos presentados, lo que reduce las posibilidades de enriquecimiento de la competencia oral de los escolares, al dejar de lado la capacidad de crear en forma propia, coherente, precisa y creativa argumentos, explicaciones, descripciones, entre otros. En este sentido, uno de los componentes lingüísticos que el estudiante debe desarrollar para la producción autónoma de textos orales y escritos, es el 
léxico, el cual debe implementarse con variedad y precisión. De ahí el interés, de este estudio, por describir las destrezas orales que presentan los niños en cuanto al nivel léxico, al producir textos orales explicativos y narrativos.

Los datos que se presentan a continuación corresponden a la investigación La competencia en la expresión oral de escolares en Costa Rica. El componente léxico desarrollada por Araya (2011), autora de la presente publicación, en la que se analizó el componente léxico, en cuanto a índice de diversidad léxica, comportamiento de categorías gramaticales y precisión léxica. Particularmente, en este artículo, se presentan los resultados obtenidos en cuanto a precisión léxica y el análisis de la diversidad léxica de las categorías gramaticales (sustantivo, verbo y adjetivo) en las producciones orales antes mencionadas.

\section{Fundamentos teóricos}

\subsection{El desarrollo de las habilidades comunicativas}

La competencia comunicativa, la cual plantea un cúmulo de conocimientos lingüísticos y de habilidades comunicativas que se adquieren a lo largo de las experiencias de socialización, nos permite consolidar que el aprendizaje de la lengua va más allá del dominio gramatical. Dicha posición se fortalece con el concepto de competencia comunicativa ofrecido por Cassany i comas et al. (1994), la cual se entiende como “(...) la capacidad de usar el lenguaje apropiadamente en las diversas situaciones sociales que se nos presentan cada día" (p. 85).

De esta manera, las convenciones lingüísticas y comunicativas son la base de la estructura comunicativa para crear y mantener el discurso; pero no sólo implican el código lingüístico sino también el qué decir, a quién y cómo. La percepción de las habilidades y los conocimientos de la competencia comunicativa es plausible en situaciones de interacción en la que hay producción del discurso.

La posición de Martínez del Río et al. (2002), en cuanto a la perspectiva comunicativa, remite al hecho de que se trabaja con la norma agregando a esta el aspecto funcional: para desarrollar una labor con las diversas dimensiones textuales es necesario trabajar con estrategias que permitan considerar los usos comunicativos en situaciones y tiempos reales. 
Se necesita que los estudiantes desarrollen habilidades para participar en el proceso de crear y comunicar a partir de las palabras. En el aula, lo anterior equivale a saber leer textos diversos; generar resúmenes o reseñas y saber sus diferencias con base en sus propósitos; escuchar sólo para interactuar con el otro o para analizar un determinado texto; hablar para conversar, exponer o persuadir. Fuera del ámbito estrictamente escolar, crear con las palabras es producir un recado o leerlo, solicitar información en una determinada dependencia, escribir una solicitud de empleo, entre otros.

Por lo tanto, el acto comunicativo es graduado según las intenciones y las necesidades del emisor. Determinar la situación comunicativa y el emisor al cual va dirigido el mensaje, las habilidades y los conocimientos lingüísticos indispensables para adecuar el mensaje con vocabulario diverso y preciso, son competencias que el infante va adquiriendo a partir de una serie de experiencias vividas -a través de los años- en su entorno cotidiano y familiar, no obstante, es necesario estimularlas para que alcancen su máximo nivel de desarrollo.

De este modo, la adquisición de la competencia comunicativa se desarrollará progresivamente a lo largo de las etapas del dominio lingüístico y de ahí la necesidad de acceder a descripciones de los grados transitorios e intermedios del largo proceso de la adquisición y perfeccionamiento lingüístico.

Al respecto, Canale, 1983, señalado por Mendoza Fillola (2004, p. 48),

(...) determina la pertinencia de la competencia gramatical (dominio del código lingüístico), de la competencia sociolingüística (capacidad para adecuar el mensaje al contexto), de la competencia discursiva (capacidad de formar enunciados coherentes) y de la competencia estratégica (capacidad de resolver problemas comunicativos).

Particularmente, cada una de estas subcompetencias se encuentra integrada por una serie de saberes lingüísticos y no lingüísticos que serán adquiridos y desarrollados según el grado participación de las personas en procesos comunicativos reales y en las cuales la presencia del léxico en forma diversa y precisa es un insumo indispensable para lograr un acto comunicativo adecuado. 
En este proceso, la escuela se convierte en un espacio estratégico para ampliar la competencia comunicativa, ya que se adquieren y desarrollan nuevos registros y códigos con los que el alumno puede involucrar conocimientos y habilidades para construir una comunicación más eficaz.

\subsection{Niveles lingüístico-textuales del discurso oral}

El análisis del discurso nos permite determinar características y prácticas discursivas diversas, desde situaciones comunicativas informales y espontáneas, hasta las más formales que requieren de una planificación. El uso de la palabra deberá adecuarse a la situación comunicativa para responder al contexto en que se presenta. Calsamiglia y Tusón (2008) caracterizan los diversos niveles lingüístico-textuales del discurso oral, en los que el vocabulario como "insumo básico" para la producción de los textos requerirá un respectivo tratamiento:

- Nivel fónico: Se refiere a la pronunciación y la prosodia (entonación de los diferentes enunciados interrogativos y exclamativos, marcar el énfasis en una frase, distinguir los ritmos y grupos tonales tales como las pausas de ritmo lento y relajado).

- Nivel morfosintáctico: En el caso de la producción oral planificada, responde a patrones retóricos elaborados dado que la complejidad es más elaborada en el plano sintáctico. La producción oral más espontánea presenta frecuentes discordancias, repeticiones, uso de "muletillas", utilización de la yuxtaposición y la coordinación para relacionar oraciones e incluso alteraciones sintácticas que se van resolviendo en el momento de la conversación. Las subordinaciones son menos utilizadas.

- Nivel organizacional y discursivo: Este aspecto se refiere a la estructura del discurso: inicio, progresión informativa y finalización del mismo. Dentro de la organización del texto se observan marcas de coherencia tales como marcadores discursivos, conectores y marcas de interacción.

- Nivel léxico: En relación con el código oral, la variación léxica sirve para marcar el tono de la interacción y las finalidades y motivos que se persiguen. También puede indicar características socioculturales y ser marcador de pertenencia a un grupo. El bajo grado de densidad léxica y el alto grado de redundancia están presentes como características del discurso oral (considerando el grado de espontaneidad de la interacción 
comunicativa), por lo tanto, se pueden visualizar repeticiones, imprecisiones léxicas, paráfrasis, deícticos, proformas léxicas, frases hechas y expresiones idiomáticas

De los niveles anteriores se detalla a continuación una descripción de los elementos que conforman el nivel léxico, específicamente la diversidad y precisión léxica, las cuales serán objeto de análisis en los textos orales que conforman el corpus de esta investigación.

\subsubsection{Descripción del nivel léxico en cuanto a diversidad y precisión léxica}

El léxico va más allá de un conjunto de palabras de una lengua, es decir, un simple caudal de vocabulario. Es más bien un todo interrelacionado cuyas reglas permiten la creación de nuevas formas.

De este modo, en la comunicación oral el niño no sólo incorpora vocabulario, sino que lo utiliza dependiendo de la situación y de los interlocutores; por lo tanto, la adquisición y el desarrollo de las palabras implican el reconocimiento del componente acústico, el dominio de significados, el empoderío de reglas morfosintácticas y las distintas estrategias para la formación de palabras. De ahí que estas habilidades se develan como competencias de la oralidad, en este caso del nivel léxico, con implicaciones en las otras competencias del discurso oral.

La variedad léxica, la cual es definida como la diversidad de palabras de mayor contenido léxico (sustantivos, verbos y adjetivos) que usan los niños, en producciones textuales de 50 enunciados. Murillo Rojas (2011) permite determinar la destreza que debe poseer el hablante respecto de la construcción de textos orales, que además no sólo implica un amplio bagaje de vocabulario sino también las reglas sintácticas que rigen las categorías y subcategorías gramaticales de las palabras empleadas en el discurso. La descripción del número de distintas palabras utilizadas adecuadamente en la producción oral nos permite visualizar que la competencia léxica exige un proceso creativo por parte del usuario de la lengua; la creación del mensaje no solamente responderá a las normas gramaticales y de organización del discurso puesto que debe responder también al componente pragmático (en cuanto a la finalidad y la intención que se pretende transmitir en el discurso). 
Por su parte, Mendoza Fillola (2004) menciona que las competencias a nivel léxico se incrementan con la cantidad y calidad de estímulos recibidos, por ende, el dominio del vocabulario requiere de estrategias adecuadas para conocer las palabras, sus significados y usos. En correspondencia, el nivel léxico - en el discurso oral - implica que el individuo domine los aspectos de pronunciación, morfológicos, sintácticos, pragmáticos, semánticos y sociolingüísticos, en otras palabras, se requiere una competencia comunicativa adecuada en esta área.

Asimismo, es evidente que estas capacidades no solo residen en el dominio de una lista de palabras, sino en la interrelación que el individuo establece entre estas para la producción del texto oral que responda y se adecue eficazmente a una situación comunicativa determinada. De ahí que la precisión léxica conceptualizada como:

El uso de las palabras con la mayor propiedad y precisión posible, es decir, con su significado o sentido peculiar, de manera que exista una correspondencia biunívoca entre dicho significado y el concepto que con ellas se quiere expresar (propiedad léxica); asimismo, hay que usarlas con concisión y exactitud rigurosa, es decir, expresando los conceptos que significan con brevedad y economía de medios. Carratalá Moreno (2006, p. 1)

Se establece como una competencia que permite la construcción de textos orales y escritos, adecuados a una intención de significado que se quiere expresar en forma concisa y precisa. El vocabulario debe enseñarse tanto en amplitud como en exactitud. La variedad y riqueza léxica permite al hablante adaptarse mejor a un tema y participar, con más certeza y fluidez, en la interacción comunicativa. El aprendizaje del vocabulario debe partir de las mismas temáticas de los programas de estudio y de situaciones que sean significativas para los estudiantes. Este nivel podrá ampliarse mediante el uso de campos semánticos, la formación de nuevas palabras a través de estrategias de derivación, mediante la comprensión de significados y del uso pertinente del vocabulario en un contexto adecuado. Al respecto Nippold (1998) sugiere trabajar la amplitud, la definición y las relaciones de las palabras dentro de contextos significativos, en este caso utilizado el razonamiento inductivo mediante las analogías, en las cuales se realizan comparaciones entre conceptos y por otro lado, el razonamiento deductivo a través de los silogismos, donde se relacionan dos proposiciones 
y se establecen inferencias o conclusiones de ambas. Igualmente se rescata en el aula el trabajo didáctico de la metáfora y la símil.

\subsection{Textos narrativos y explicativos}

Con respecto a los diferentes tipos de textos que los usuarios de la lengua producen, Calsamiglia y Tusón (2008) señalan los siguientes: narrativo, descriptivo, explicativo y argumentativo. Asimismo, de acuerdo con las evidencias encontradas por Barboza Brenes et al. (2008) con respecto a la producción de textos, demuestran que los escolares -al escribir- producen mayoritariamente textos explicativos y narrativos. Por lo tanto, en este estudio interesa conocer el comportamiento de estos textos a nivel oral, con respecto a la diversidad de categorías gramaticales y la precisión léxica.

A continuación se señalan las características específicas de los textos narrativos y explicativos, de acuerdo con lo propuesto por Calsamiglia y Tusón (2008)

- Texto narrativo: Presenta una sucesión de acontecimientos en un tiempo que transcurre y se establecen relaciones causales entre los acontecimientos. Se da un proceso integrador dado que a partir de una situación inicial se llega a una situación final a través del proceso de transformación.

- Texto explicativo: La explicación parte de que hay una información previa. Los datos que se suministren del tema provienen de la propia experiencia o por la vía de la reflexión. En este sentido, la información organizada y sistematizada es parte del conocimiento producido en una cultura. La información se almacena en la memoria, por tanto, cada persona posee un conocimiento llamado enciclopédico o acumulativo que aumenta a lo largo de la vida y es usado para comunicarse con los demás.

En este sentido, la producción textual variará, respecto de su estructura y función, ya que deberá adaptarse al ámbito de uso. Por lo tanto, el vocabulario que se implemente deberá ajustarse a los modos de organización del discurso y usarse en forma precisa y coherente, con el propósito de dar respuesta a una situación comunicativa específica. 


\section{Procedimiento metodológico y análisis del corpus}

Se seleccionaron 15 estudiantes al azar, por cada nivel escolar de un centro educativo público del cantón de Montes de Oca, de la provincia de San José, con el objetivo de procesar los textos orales de 90 sujetos y desechar los textos que no cumplieran con el mínimo de 50 enunciados o más, tanto en el texto explicativo como el narrativo. Lo anterior para responder a los criterios metodológicos que se explicarán más adelante.

Al darse una constante por nivel escolar de 6 a 9 estudiantes que lograron producir ambos tipos de textos con el requisito mencionado, se escogieron 36 escolares distribuidos equitativamente (seis niños por cada grado escolar). De esta manera, se analizaron 72 producciones orales en total. Por consiguiente, la muestra fue intencionada y los estudiantes elegidos debían cumplir con la edad cronológica según el grado escolar que cursaban, no repetir el curso lectivo correspondiente, ni recibir adecuación curricular de ninguna modalidad.

Dadas las características del texto explicativo, que parte del supuesto de la existencia de información y además la almacena, organiza y sistematiza en la memoria para comunicarse con los demás, se consideró el tema: La Independencia de Costa Rica como tópico para producir oralmente y recoger los textos explicativos de los escolares que conformaron la muestra. Para ello, se formularon cuatro preguntas generadoras:

- ¿Por qué celebramos todos los años la independencia del país?

- ¿Por qué es importante ser independientes?

- ¿Cómo se celebra en su escuela y en el país la independencia?

- ¿Le gusta su país?, ¿por qué?

En el texto narrativo se da una serie de acontecimientos ocurridos en el tiempo y en el espacio; por tanto, tienen un transcurso secuencial: hay un inicio que progresa hacia un final. Para producir el texto narrativo, primero se presentó la película Cachorros en el espacio, producida por los Estudios Disney Movies. 
Para conocer el comportamiento de las categorías gramaticales (sustantivos, verbos y

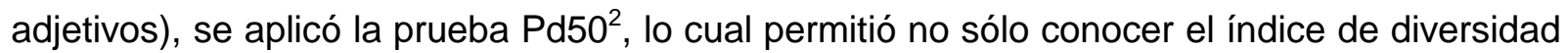
léxica, sino también cuantificar y clasificar las categorías gramaticales. Posteriormente, el uso de las categorías gramaticales se analizará de acuerdo con la precisión que se presentan en el discurso.

En este sentido, se siguieron los pasos metodológicos propuestos por Murillo Rojas (2011):

- Transcripción grafemática de las producciones textuales de los niños (realizada con la mayor fidelidad).

- División del texto en enunciados oracionales.

- Para el cálculo de número de palabras distintas, se contabilizó solo una vez cada lexema sustantivo, adjetivo y verbo. Por ejemplo: casa-casita-casilla, cuentan como un único vocablo- tipo "casa". Se considerará como lexema la parte de la palabra que no varía y que contiene su significado, en este caso: deport-e, deport-ivo, deport-istas, cuyo vocablo-tipo es "deporte".

- Respecto del número de verbos distintos, las formas infinitivas de cada expresión verbal se cuentan solo una vez. Las perífrasis verbales, las cuales son la unión de dos o más formas lingüísticas que funcionan como una unidad verbal, se contarán como un solo verbo.

- En el caso de los sustantivos distintos, se cuentan los lexemas de cada nombre una sola vez. No se consideraron los nombres propios, ya que estos pertenecen a la categoría de los sustantivos, por ejemplo: Buda, corresponde al nombre de un perro, en este caso la palabra "perro" se contabiliza como sustantivo.

- En cuanto a los adjetivos distintos, se cuentan los lexemas de cada adjetivo solo una vez

- El cálculo del número total de palabras de contenido léxico de cada texto se obtuvo al contar todas las formas de las palabras. Por ejemplo: casa-casilla-casita son tres palabras.

\footnotetext{
${ }^{2}$ Para evaluar la riqueza léxica se utilizaron muestras de textos orales de 50 enunciados. Tal como lo plantea Murillo Rojas (2011) en el Test de vocabulario para preescolares costarricenses de 3 a 6 años TEVOPREESC, con el cual se puede conocer la riqueza léxica de los escolares mediante la descripción de la variabilidad de palabras de contenido léxico tales como sustantivos, adjetivos y verbos.El cálculo del Pd 50 (prueba para valorar la riqueza léxica en cincuenta enunciados) se obtiene de la siguiente manera: número de palabras diferentes de contenido léxico entre el número total de palabras de contenido léxico
} 


\section{Resultados}

En el desarrollo de este apartado se describen los resultados concernientes al análisis del corpus según el comportamiento de los sustantivos, los verbos y los adjetivos y la precisión léxica en textos orales narrativos y explicativos.

\subsection{Descripción de categorías gramaticales}

En el caso del texto oral narrativo, las palabras diferentes en el primero y segundo ciclos se encuentran distribuidas de la siguiente forma:

Tabla 1. Distribución de categorías gramaticales. Texto oral narrativo

\begin{tabular}{|c|c|c|c|}
\hline Ciclo & Sustantivos & Verbos & Adjetivos \\
\hline Primer & 458 & 529 & 103 \\
\hline Segundo & 495 & 540 & 121 \\
\hline Total & 953 & 1069 & 224 \\
\hline
\end{tabular}

Fuente: Elaboración propia

De esta manera, se puede visualizar que en este tipo de discurso, el verbo es la categoría gramatical más utilizada por los niños de primer y segundo ciclos, seguido por el sustantivo en escasa diferencia. En cuanto a los adjetivos, la cantidad dista significativamente del verbo y del sustantivo.

Por otro lado, en el texto oral explicativo, el sustantivo ocupa el primer lugar, seguido del verbo, e igualmente la cantidad de adjetivos es minoritaria. Tal como se ilustra en el siguiente recuadro con respecto al número de palabras diferentes presentes en el texto explicativo:

Tabla 2. Distribución de categorías gramaticales. Texto oral explicativo

\begin{tabular}{|c|c|c|c|}
\hline Ciclo & Sustantivos & Verbos & Adjetivos \\
\hline Primer & 556 & 467 & 191 \\
\hline Segundo & 546 & 476 & 194 \\
\hline Total & 1102 & 943 & 385 \\
\hline
\end{tabular}

Fuente: Elaboración propia 
Al comparar los resultados obtenidos por cada tipo de texto, se identifica que la mayor cantidad de sustantivos se encuentran en el texto oral explicativo, y el mayor conjunto de verbos está en el texto oral narrativo. Por otro lado, la cantidad más representativa de adjetivos aparece en el texto oral explicativo.

Es importante resaltar que en el caso de los sustantivos más utilizados sobresalen los concretos, los cuales posiblemente al denominar un referente temporal-espacial, son más representativos para los estudiantes. Asimismo, los verbos más utilizados en las producciones orales narrativas corresponden a verbos dinámicos, los cuales se evidenciaron en los siguientes ejemplos: /me duele la cabeza /y /la gente se divierte/. Por otro lado, la inserción de verbos copulativos ser y estar, como en las expresiones: /soy tico/ y /estaban contentos/. Asimismo, en los textos orales explicativos sobresalieron los verbos estativos, como por ejemplo: /mi papá conoce de la independencia/ y /yo vivo en Costa Rica/. Por último en el caso de los adjetivos los más frecuentes son los calificativos, los cuales en la mayoría de las ocasiones aparecen junto a los verbos copulativos "ser y estar" o aparecen unidos asindéticamente al sustantivo o nombre.

Murillo Rojas y Sánchez Corrales (2002) ya habían acotado que el sustantivo es la categoría gramatical más numerosa del español y es la que se aprende con mayor facilidad, lo que responde a los datos obtenidos en esta investigación, donde los nombres son los más producidos al ser contabilizados en ambos tipos de textos orales, es decir, se registraron 2055 sustantivos y por otro lado, 2012 verbos en total, aunque realmente la diferencia no es abismal, como si ocurre con respecto a los adjetivos, que en total se produjeron 609 únicamente, lo que asiente reflexionar sobre el abordaje didáctico de esta categoría gramatical.

De acuerdo con Murillo Rojas (2001):

Los adjetivos son palabras que se aplican a otras palabras que nombran objetos físicos o mentales. Más específicamente un adjetivo modificador adscribe propiedades cuya especificación sirve para definir o delinear con mayor precisión a la entidad mentada, para caracterizarla e identificarla, entre varias similares, para clasificar y establecer taxonomías culturales y científicas. (p.3) 
Por lo tanto, es claro que la producción y el uso del adjetivo requiere de una operación o actividad más precisa y compleja del pensamiento, lo que permite dar exactitud a la idea que se construye. De ahí la pertinencia del trabajo didáctico de esta categoría gramatical, con el propósito de mejorar el enriquecimiento léxico y a la vez que el hablante concrete y precise más su producción, pues la pertinencia del adjetivo va más allá de aquella palabra que opcionalmente puede aparecer en un enunciado oracional.

De acuerdo con lo señalado por Barboza Brenes, G. et al. (2008), la secuencia textual narrativa presenta una sucesión de acontecimientos en un tiempo que transcurre y se establecen relaciones causales entre los acontecimientos, y, por otro lado, la explicativa parte de una información previa, la cual proviene de su propia experiencia y cultura.

Lo antepuesto se refleja en los repertorios léxicos registrados, ya que en el caso particular del texto oral narrativo se produjeron más verbos que sustantivos y adjetivos, lo cual permitió reflejar una característica típica de este tipo texto, al centrarse principalmente en el relato de un ciclo o proceso de acciones; por otro lado, el texto oral explicativo producido, presenta más sustantivos, pues es evidente que este tipo de secuencia textual requiere primordialmente de la intervención de información (conceptos e ideas enmarcadas dentro de una cultura), la cual almacena el sujeto, para ser usada en un momento específico.

Esta última apreciación, permite asentar la necesidad de reformular la intervención de la didáctica del texto en las aulas escolares, pues el tratamiento del léxico en función de las características particulares de cada secuencia textual, incide satisfactoriamente en la amplitud, la precisión, la coherencia y la cohesión de las producciones, tanto en el plano oral como el escrito.

\subsection{Precisión léxica}

Con el propósito de analizar el comportamiento de la precisión léxica en los textos orales producidos por los sujetos de este estudio, se hace una descripción de ejemplos que corresponden a imprecisiones en el uso del vocabulario, en las categorías gramaticales de sustantivos, verbos y adjetivos. 
Se utilizaron como base de consulta el Diccionario de la Lengua Española (en línea www.drae.com.), el Diccionario combinatorio del español contemporáneo de Bosque Muñoz (2004) y los diccionarios de costarriqueñismos de Agüero Chaves (1996) y Quesada Pacheco (2007).

En términos generales, según los resultados obtenidos de la precisión léxica de los textos orales narrativos y explicativos, se pueden derivar las siguientes conclusiones:

Con respecto a la categoría gramatical de los sustantivos:

- El uso de términos imprecisos con respecto a la categoría gramatical de los sustantivos, tales como: "cosa" "algo" "todo" y "lugar" sobresalieron tanto en los textos orales narrativos como explicativos. En este sentido, la mayoría de las imprecisiones consisten en el empleo de la palabra "cosa", la cual tal como indica Sánchez Avendaño (2005, p. 2) denota "un bajo nivel de informatividad y explicitud, que limita la comprensión de lo que se habla según sea el contexto desarrollado en cada producción". Esta situación fue evidente en trece escolares. Algunos ejemplos que fundamentan lo expuesto son los siguientes:

- todo el mundo se fue a ver otras cosas de la estación y nadie los vio los perros se empezaron a poner los trajes espaciales

- los perros se fueron a la nave para conocerla por dentro y ver que era la cosa y después querían salir

- dijo cosas verdad que debía hacer para regresar y ellos iban haciendo el viaje de regreso

- que les explicaban los guías de la estación espacial entonces los niños preguntaban cosas a los guías sobre el espacio

- desviarlo a la lluvia de cosas

- atravesaron muchas cosillas y cosotas eh

- pasan por un montón de cosas hasta que llegan a la Tierra

- casi se estrellan por unas cosotas que dañaron a la nave y diay una antena que se torció pero que un perrito salvó

- empezaron a caer chispas de cosas de arriba y entonces la nave del loco explotó

- las cosas feas han quedado atrás en los tiempos de antes

- hay no que me impongan otras cosas amigos que dicen cosas

- ya nos han impuesto cosas

- también se vende café y cosas de maíz 
- que nos vengan a decir cosas que debemos comer o así

- las personas pueden expresar muchas cosas pero hay países grandes y pequeños

- uno no puede decidir cosas malas

- no hay tantas reglas o algo así o sea no se pueden hacer ciertas cosas en países porque hay reglas

- sí yo hago cosas buenas si... cosas buenas por el país

Por otra parte, las palabras "cosa" y "cosas" son utilizadas como estrategia discursiva de cohesión, con una frecuencia de doce veces en las producciones analizadas. En los siguientes casos, dichos términos son especificados en forma parcial o completa en el contexto del discurso por los escolares en forma pospuesta o antepuesta:

- pasó una estrella fugaz y ellos desearon cosas que querían ir a la luna el perrito deseó mucho que el deseo se cumpliera

- fueron conociendo las cosas de las estación espacial y ahí ellos conocían las cosas y vieron luces y palancas

- el ruso al darse cuenta de las cosas este que había tantos perros eh los quiso atrapar

- el señor que había quitado la gasolina volvió hacer cosas malas y alteró la ruta buena y los mandó a una ruta de meteoritos y ellos se fueron por ahí

- querían saber cosas verdad cómo tiraban las naves al espacio

- destruyó la antena que tenía conexión con la estación para salvar a los cachorritos y entonces la zarigüeya les dijo cosas que si la podían este no ella les dijo una cosa después este que enviaran a un cachorrito que la arreglara

- para mí la película se trata de varias cosas este bueno que si uno tiene un sueño hay que lucharlo y poderlo lograr como el perrito ruso que quería regresar a la Tierra con su dueño

- los de la estación espacial les dijeron ahí cosas que harían todo lo posible y que los perros volvieran a la Tierra

- a mí no me gustaría vivir en un país con muchas cosas feas como esclavitud

- podemos disfrutar de muchas cosas bonitas como la playa, la comida, la escuela, la familia y muchas cosas más

- que con la independencia Costa Rica pudo tener muchas cosas importantes como el voto

Por otra parte, el término "todo" identificado presente en los textos interfirió en la comprensión de los mismos, pues no se especifica a lo largo de la producción el referente de la palabra. Estos son algunos ejemplos: 
- lo mandaron a conectar la antena y todo

- se subieron a las sillas y veían todo desde el espacio

- y entonces ah la zarigüeya que podía hablar el idioma de perros le dijo al perro que todo se lo comiera que debía arreglar la nave y él lo logró

Igualmente, se identificaron otros términos imprecisos que conllevan el uso incorrecto de información. En este caso, es evidente en algunos escolares el poco dominio de datos históricos referentes al acontecimiento que ha trascendido a lo largo del tiempo en nuestro país, en este sentido "la independencia", la cual relaciona elementos culturales, políticos y sociales, que por lagunas en su erudición ocho escolares no los contemplan en sus producciones:

- Costa Rica se independizó de Nicaragua

- nosotros nos independizamos de Nicaragua y Guanacaste

- nos independizamos de Nicaragua y Guanacaste

- ah la independencia recordamos a Juan Santamaría que quemó el mesón

- Costa Rica se independiza de Guanacaste... no me acuerdo bien de quien bueno de Nicaragua

- los de Guanacaste se vinieron para Costa Rica bueno los del Partido de Nicoya este ah este bueno Costa Rica antes de la independencia pertenecía a Guatemala

- independizamos de un país no sé cuál es creo que es Nicaragua

- yo pienso que el Partido de Nicoya se independizó de Costa Rica

Por otro lado, nueve escolares se refieren en forma imprecisa al término "meteoritos", los cuales golpean a la nave en el transcurso de la película. Los ejemplos son los siguientes:

- después cuando iban a regresar a la Tierra tuvieron que batallar una lluvia de chunches. En este ejemplo también es impreciso el verbo, lo adecuado sería "enfrentar".

- los perros vieron muchos chunchillos pesados y se rompió la antena

- se toparon con una lluvia de rocas

- se toparon con una lluvia de roca

- que mandó a la nave donde una batalla de asteroides

- la zarigüeya les dijo de enfrentar una tormenta de piedras

- lo lograron sobretodo con la lluvia de piedras

- la antena que una piedra dobló 
- batallaron con una vara de chunches. En este ejemplo se utiliza una forma verbal imprecisa, que en este caso lo adecuado sería "enfrentar", además aparece el término coloquial "vara" y "chunche".

En relación con el uso del costarriqueñismo "chunche", el cual es definido como "objeto, cosa, en realidad cualquier cosa" (Agüero Chaves 1996, p. 123 y Quesada Pacheco 2007, p.129), es empleado para referirse al término nave espacial:

- un perro usó su propio gas para arreglarla el chunche ese

- el ruso pudo escapar en una chuncha especial que tenía su propia estación

- los perritos lograron aterrizar bien en el chunche... ese esa cosa

- los perritos lograron aterrizar bien en el chunche ese esa cosa

- que los llevó hasta una nave espacial entonces se fueron en un avión este a a la luna En este ejemplo se confunde el término "avión“ por "nave espacial".

Por otro lado, aparecen las formas imprecisas de la palabra "chunche":

- diay se me fue la cosa el chunche ay no

- por eso la maestra nos dice limpien chiquitos los chunches

- en la tele yo he visto guerras con chunches

Cabe destacar que el término "chunche", no se registra en el DRAE y ni en el Diccionario combinatorio de Bosque Muñoz (2006).

Asimismo, aparecen los siguientes costarriqueñismos en las expresiones elaboradas por los niños:

- las personas se pueden pelear con mecos

- nos comimos un apretado

- mi mamá dice que hay ladrones o pintas y se roban a los chiquitos en las calles

- le caen a uno las pintas

- puedo ir al chante de mis amigos.

- las güilas quedarían embarazadas muy jóvenes en la escuela.

- dice que parezco un pachuco

- me da dolor de panza y como cuiteadera

- roban por piedra a la gente 
Como creaciones léxicas sobresale el uso del término "pitufo" o "pitufote" para la palabra "pedo", "flatulencia" o "gas", el cual no es registrado por los diccionarios de costarriqueñismos y el DRAE:

- se echaba unos pitufos

- el perro más comelón de frijoles se tiró un pitufote

- que el perro gordo se chochó un pedo

Con respecto a estos ejemplos, palabra "pitufo" no se encuentra definida en el DRAE y los diccionarios de costarriqueñismos.

De acuerdo con el contexto en que aparece la palabra, las definiciones aceptables serían las pertinentes a gas, pedo, flatulencia o ventosidad.

Igualmente sucede con el uso de la forma "chochó", la cual no concuerda con el contexto de uso. De acuerdo con Quesada Pacheco (2007, p. 125) el término se refiere a "una persona que no está en sus cabales o un automóvil que no funciona" y, por otro lado, el DRAE lo define como con facultades mentales débiles o el expresar cariño a otras personas. En el Diccionario combinatorio del español de Bosque Muñoz (2006) se hace mención de la combinación del verbo chochear ante sustantivos y adverbios.

En cuanto a la categoría gramatical de los verbos:

El verbo "hacer" fue utilizado frecuentemente por los escolares en diversos contextos, en este sentido, en la mayoría de los casos se presentó como "hacer cosas", lo que generó imprecisión en las ideas. Esta situación se presentó particularmente en los textos orales explicativos, respectivamente veintidós contextos. En este caso es utilizada por los hablantes como un comodín, para expresar palabras o situaciones determinadas que estos no llegan a precisar de acuerdo con el contexto en uso, por ejemplo en la frase: podemos hacer cosas, puede referirse a: proponer-crear-aprobar-presentar leyes, de acuerdo con la progresión temática del texto y en general del contexto

Los siguientes ejemplos tomados de los textos fundamentan lo anterior: 
- la independencia hace felicidad

- hace sus cosas y no lo que lo manden

- no se hacen cosas feas en la calle y bueno la gente se porta bien ah... sí ayudan a la gente mayor en las calles y no roban y hacen cosas buenas por los demás

- hacemos muchas cosas

- nadie nos obliga hacer cosas malas no queremos

- y podemos hacer nuestras cosas

- pueden hacer cosas sin miedo

- hacerse cosas que uno quiere

- había que pedir permiso para hacer las cosas de este país

- las maestras explican cosas en las aulas y diay que en Costa Rica las personas pueden hacer muchas cosas

- eh no nos quiere dejar hacer cosas

- hacer cosas y nadie me hace nada malo bueno hay ladrón

- es y hacen cosas feas

- a uno lo obligan a hacer las cosas impuestas aunque no nos gustan

- podemos hacer cosas para nosotros

- no éramos independientes más bien ahora sí y podemos hacer las cosas con independencia pero eso sí no se puede matar o tampoco se hacen cosas malas como los robos

En el caso del verbo "tirar" se emplea de forma imprecisa en los contextos en que aparece. En este caso, la forma verbal más precisa corresponde a "lanzar". Esta situación se presenta en:

- verdad cómo tiraban las naves al espacio

- ellos quería ver cómo tiraban una nave a la luna

- las personas que manejan la nave la tiran al espacio

- ahí tiran naves espaciales

Por otra parte, dentro de las construcciones verbales realizadas por los escolares se encontraron problemas de conjugación en ocho ocasiones, como en los siguientes ejemplos:

- no podieron ir a una excursión espacial al principio

- se los punieron

- que le ponieron a cada perro

- ponieron la ropa

- una zarigüeya les dijo que tenieran cuidado con los meteoritos 
- se montaron en una camioneta escolar con los bultos revolvidos

- que estaban revolvidos en los bultos

En estos ejemplos se presenta una incorrecta construcción de la palabra. Se produce un proceso de creación morfológica de conformidad con el proceso de adquisición del vocabulario, como con el patrón regular temer - temido / revolver - revolvido, entre otros, característica del hablante en su proceso de adquisición de la variedad prestigiosa de la lengua.

Con respecto a las formas verbales generadas con el término "chunche" se transcribe dos ejemplos:

- chuncherequearon la antena y la puso bien

- que una piedra la chuncherequeó

En relación con la categoría gramatical de los adjetivos:

Los escolares recurren a estos adjetivos genéricos, tales como en el empleo de feo/bonito y bueno /malo y no producen otros que expresen características más específicas, que describan mejor lo valorado, pues se carece de un repertorio léxico mayor para indicar cualidades como por ejemplo: agradable, precioso, bello, desagradable, entre otras.

- eso es feo

- la esclavitud es fea

- es feo que nos mandaran

- que feo verdad

- sería feo

- que feo verdad

- es feo estar en la cárcel

- eso es feo

- imagínese sin libertad sería feo bien feo

- se gritan cosas feas

- también la personas hacen cosas feas entonces no sólo el gobierno por ejemplo o la naturaleza y todos somos responsables

- otras playas de otros lugares son feas

- gracias a la celebración de la independencia somos un país bonito 
- es muy bonito

- así no dependemos de nadie de nadie con malas ideas

- eso puede ser malo

- dejó muchas huellas y cosas buenas

- eso es algo no bueno

Asimismo, la mayor parte de los adjetivos producidos por los estudiantes, se refieren a términos o palabras consideradas como costarriqueñismos, frases hechas, metáforas y préstamos dialectales.

En los siguientes ejemplos se describen adjetivos considerados como costarriqueñismos:

- y vieron los controles y los planetas de largo y vieron los controles y los planetas de largo

- los perros seguían observando de largo

- veían a la Tierra de largo

En estos ejemplos lo correcto es el uso del vocablo "lejos", pues "largo" es un adjetivo en función adverbial.

En los consecuentes casos se presenta el costarriqueñismo "colados" para caracterizar a los sujetos participantes en la trama de la película. Dicho término se conceptualiza como aquella persona que está involucrado en un asunto (Agüero Chaves 1996, p. 69 y Quesada Pacheco 2007, p. 136). EI DRAE hace referencia a dicha acepción, no así el Diccionario combinatorio del español de Bosque Muñoz (2006).

- ellos se montaron de colados en la nave

- se metieron en el bus cuando nadie se dio cuenta y se fueron de colados a la excursión

El uso de "soplas", definido como "soplón/delator" (Quesada Pacheco 2007, p. 364) es utilizado por un escolar para caracterizar la actitud de uno de los perros en la trama de la película. Bosque Muñoz (2006) no registra esa forma como tal, en este caso señala "soplo de" combinado en contextos ante sustantivo. EI DRAE si considera dicha acepción.

- el perro se va de soplas a contarle a los demás perros 
Igualmente el término "chiva" que dentro de sus acepciones contempla el significado de la palabra como "algo bonito, alegre y elegante" (Agüero Chaves 1996, p. 73 y Quesada Pacheco 2007, p. 124) aparece en cuatro contextos para indicar agrado:

- este para mí fue una película súper chiva

- la película estaba chivísima

- la película súper súper chiva que habían cinco perritos muy traviesos

- los perros desfilaron con sus dueños en unas naves chivas

Esta acepción de "chiva" no se registra en el DRAE ni en el Diccionario combinatorio del español de Bosque Muñoz (2006):

Por otra parte en las producciones se hallaron las siguientes frases coloquiales que caracterizan situaciones o hechos:

- qué torta porque los chiquitos no sabían

- se tuvieron que hacer los machos

- que sea tonto Esta situación responde a una frase coloquial costarricense, que se refiere a "qué barbaridad" "demasiado" "cruel", entre otras. La expresión "que sea tonto", se ha usado en vez de "sea tonto", lo que evidencia que el niño no ha logrado una adecuada adquisición de su uso.

- sin querer queriendo por traviesos se fueron por una dirección. En este ejemplo se visualiza un préstamo dialectal de la Serie del Chavo del Ocho, por tanto es un mexicanismo de origen.

El término "polo" aparece en distintas construcciones, con el propósito de caracterizar los participantes de los textos producidos, tales como:

- que eso es polo

- son polos pero mi mamá dice que no

- comemos tamales con café huy que polo

Quesada Pacheco (2007, pp. 294-322) define dicho costarriqueñismo de la siguiente forma: Despectivo, de mal gusto. Dicha acepción no se registra en el DRAE ni en el Diccionario combinatorio del español de Bosque Muñoz (2006).

De acuerdo con los resultados anteriores, en forma general, se identificaron una serie de estrategias discursivas utilizadas para los hablantes, con el propósito de construir producciones orales adecuadas a un contexto comunicativo. En este sentido, los estudios 
sobre la norma ofrecen la oportunidad para planificar los distintos aspectos de la educación lingüística desde una perspectiva racional, lejos de la actitud "purista" y "permisiva".

Por otro lado, como parte de la cultura, los estudiantes proporcionaron en sus producciones orales diversos costarriqueñismos, los cuales no se consideraron como imprecisiones, pues forman parte de la identidad cultural de los escolares.

La mayoría de los costarriqueñismos se encuentran incorporados en el DRAE, pero otras palabras no se encuentran o el significado que plantea, como por ejemplo en las palabras chochear y pitufo, las cuales no son ajustables al contexto. De ahí la importancia de la escuela como orientadora en el trabajo del léxico de los escolares.

\section{Conclusiones}

En cuanto a las categorías de palabras (sustantivos, verbos y adjetivos) que fueron analizadas en esta investigación, se encontró que los adjetivos son los menos producidos, lo cual implica una ineludible transformación de la mediación pedagógica en el aprendizaje de la lengua en la escuela, dejando de lado el enfoque gramatical tradicional, y, por el contrario, optar por otro más productivo y adecuado, que permita al escolar el uso del adjetivo en situaciones comunicativas funcionales, lo que no solo mejorará la variedad del léxico sino también una producción de enunciados más concretos y precisos.

En relación con la precisión léxica, las palabras "cosa", "chunche", "hacer cosas", "feo/bonito" y "malo/bueno" son claros ejemplos de imprecisión léxica encontrada en los textos orales narrativos y explicativos producidos por los sujetos de este estudio. Dicha debilidad puede fortalecerse con la adquisición de nuevo vocabulario, para luego ser contextualizado en textos orales de diversas temáticas.

Con el propósito de incrementar la precisión léxica, los docentes tienen un papel transcendental en la implementación de estrategias didácticas que fomenten la precisión léxica en diversas situaciones lingüísticas. De acuerdo con Alegría de la Colina (1993), se deben incorporar estrategias para trabajar el vocabulario con precisión y exactitud, entre ellas se consideran el análisis de la palabra en su contexto, la polisemia, homonimia, 
sinonimia, antonimia, lenguaje figurado, entre otras. Además que se involucre el desarrollo de las categorías gramaticales no como contenido, sino más bien como elementos que permitan al estudiante expresarse en su lengua materna en forma precisa y concisa.

Es importante resaltar que la madurez sintáctica de los niños influye notablemente en la construcción del discurso. Esta se adquiere a través de la cotidianeidad, sin embargo, las experiencias que el niño enfrenta permiten estimular el desarrollo de la misma. Al respecto, Báez Pinal (2007, p. 25) afirma que la contribución hacia esta madurez no se trata de secuenciar contenidos, tales como clases de palabras, marcas de coordinación, puntuación, entre otros, más bien su comportamiento deber ser continuo en relación con las actividades de creación de textos orales y escritos.

\section{Referencias}

Agüero Chaves, Arturo. (1996). Diccionario de costarriqueñismos (Tomo II, cuarta parte). San José: Asamblea Legislativa.

Alegría de la Colina, Margarita. (1993).Variedad y precisión del léxico. México: Editorial Trillas.

Araya Ramírez, Jéssica. (2011). La competencia en la expresión oral de niños escolares en Costa Rica. El componente léxico. (Tesis de posgrado). Universidad de Costa Rica.

Barboza Brenes, Graciela et al. (2008). La expresión escrita en la escuela primaria. (Tesis de grado).Universidad de Costa Rica.

Báez Pinal, Gloria. (2007). La enseñanza del español. Problemas y perspectivas en el nuevo milenio. México: Sociedad y Cultura.

Bosque Muñoz, Ignacio. (2004).Redes. Diccionario combinatorio del español. Madrid: Ediciones S.M.

Calsamiglia, Helena y Tusón, Amparo. (2008). Las cosas del decir. Barcelona: Ariel.

Cassany i comas, Daniel. et al. (1994). Enseñar lengua. Bacelona: Graó.

Carratalá Moreno, Fernando. (2006). Precisión y variedad léxica. Revista TIMOTEL, 10 (2), 1-5. 
Lomas, Carlos. (1999). Cómo enseñar a hacer cosas con palabras (Volumen I). Barcelona: Paidós.

Martínez del Río, María Asunción et al. (2002). Del texto y sus contextos. México: Edere.

Mendoza Fillola, Antonio. (2004). Didáctica de la lengua y la literatura. Madrid: Pearson.

Murillo Rojas, Marielos. (2001). En torno al nombre adjetivo en el discurso oral de los niños costarricenses: lineamientos para su enseñanza. Revista Actualidades Investigativas en Educación, 1 (2), 3. Recuperado de http://revista.inie.ucr.ac.cr/buscar-articulos/controlador/Article/accion/show/articulo/entorno-al-nombre-adjetivo-en-el-discurso-oral-de-los-ninos-escolares-costarricenseslineamientos-para-su-ensenanza.html

Murillo Rojas, Marielos. (2011). Test de vocabulario para preescolares costarricenses de 3 a años, TEVOPREESC. San José: Editorial de la Universidad de Costa Rica: Serie ELEXHICÓS.

Murillo Rojas, Marielos y Sánchez Corrales, Víctor. (2002). Crecimiento del léxico básico de los escolares costarricenses: código oral. Káñina, Revista de Artes y Letras de la Universidad de Costa Rica, 24(2), 97-108.

Nippold. Marylin. (1998). Later language Development. Texas: Pro-ed. An International Publisher.

Quesada Pacheco, Miguel Ángel. (2007). Nuevo diccionario de costarriqueñismos. Cartago: Tecnológica de Costa Rica.

Sánchez Avendaño, Carlos. (2005).Los problemas de redacción de los estudiantes costarricenses: una propuesta de revisión de la lingüística del texto. Revista de Filología y Lingüística, XXXI (1), 267-295. 